\title{
Triangle Formulas in the Complex Plane
}

\author{
By Philip J. Davis
}

1. Introduction. In the course of studying "simple" quadratures, the following identity was derived. Let $T$ designate a triangle lying in the complex $z$ plane whose vertices are $z_{1}, z_{2}, z_{3}$. If $A$ designates the area of $T$ and if $f$ is any function that is regular in the closure of $T$, then

$$
\begin{aligned}
\frac{1}{2 A} \iint_{T} f^{\prime \prime}(z) d x d y=\frac{f\left(z_{1}\right)}{\left(z_{1}-z_{2}\right)\left(z_{1}-z_{3}\right)}+\frac{f\left(z_{2}\right)}{\left(z_{2}-z_{1}\right)\left(z_{2}-z_{3}\right)} & \\
& \quad+\frac{f\left(z_{3}\right)}{\left(z_{3}-z_{1}\right)\left(z_{3}-z_{2}\right)} .
\end{aligned}
$$

This formula can be put in the alternative form

$$
\text { (1') } \quad \frac{1}{2 A} \iint_{T} f^{\prime \prime}(z) d x d y=f\left(z_{1}, z_{2}, z_{3}\right)=\left|\begin{array}{ccc}
1 & 1 & 1 \\
z_{1} & z_{2} & z_{3} \\
f\left(z_{1}\right) & f\left(z_{2}\right) & f\left(z_{3}\right)
\end{array}\right|:\left|\begin{array}{ccc}
1 & 1 & 1 \\
z_{1} & z_{2} & z_{3} \\
z_{1}{ }^{2} & z_{2}{ }^{2} & z_{3}{ }^{2}
\end{array}\right|,
$$

where $f\left(z_{1}, z_{2}, z_{3}\right)$ designates the second divided difference of $f$ with respect to $z_{1}, z_{2}, z_{3}$. It may also be written as

$$
R\left(f ; z_{1}\right)=\frac{\left(z_{1}-z_{2}\right)\left(z_{1}-z_{3}\right)}{2 A} \iint_{T} f^{\prime \prime}(z) d x d y
$$

where $R\left(f ; z_{1}\right)$ is the remainder at $z_{1}$ due to linear interpolation to $f(z)$ at $z_{2}$ and $z_{3}$.

This formula was subsequently found in Approximation: Theory and Practice by I. J. Schoenberg (Notes on a series of lectures at Stanford University, 1955) and correspondence with Schoenberg revealed that it was obtained by him and T. Motzkin in 1951-1952 during one of their numerous luncheon conversations. In the set of notes just referred to, the formula was derived by applying the HermiteGenocchi integral representation for divided differences, and this approach suggests certain generalizations to $n$th order divided differences.

The object of the present paper is to publicize this interesting formula, to give an alternate proof of it, and to derive a number of consequences and results related to the alternate approach.

2. Alternate Derivation of the Motzkin-Schoenberg Formula. We begin by recalling several elementary formulas. If $z_{1}$ and $z_{2}$ are two distinct points in the complex plane, then the equation of the straight line passing through them is

$$
\left|\begin{array}{lll}
z & \bar{z} & 1 \\
z_{1} & \bar{z}_{1} & 1 \\
z_{2} & \bar{z}_{2} & 1
\end{array}\right|=0
$$

Received February 26, 1964. This work was supported by the Office of Naval Research, Contract Nonr 562(36). 
or

$$
\bar{z}=\left(\frac{\bar{z}_{1}-\bar{z}_{2}}{z_{1}-z_{2}}\right) z+\left(\frac{z_{1} \bar{z}_{2}-z_{2} \bar{z}_{1}}{z_{1}-z_{2}}\right) .
$$

Formula $\left(2^{\prime}\right)$ is the simplest example of a "Schwarz reflection function" for an analytic arc, which expresses the equation of the arc in the form

$$
\bar{z}=S(z) \text {. }
$$

(See, e.g., Davis and Pollak [3, p. 6-7, 23-27].)

If $z_{1}, z_{2}, z_{3}$ are three noncollinear points, then the directed or signed area $A\left(z_{1}, z_{2}, z_{3}\right)$ of the triangle with these vertices is given by

$$
A\left(z_{1}, z_{2}, z_{3}\right)=\frac{i}{4}\left|\begin{array}{ccc}
z_{1} & \bar{z}_{1} & 1 \\
z_{2} & \bar{z}_{2} & 1 \\
z_{3} & \bar{z}_{3} & 1
\end{array}\right|
$$

(See, e.g., Deaux [4, p. 59-60].)

Green's Theorem in the plane can, with the help of the Cauchy-Riemann equations, be put in the form

$$
\iint_{B} f^{\prime}(z) d x d y=\frac{i}{2} \int_{\partial B} f d \bar{z} ; \quad d \bar{z}=d x-i d y .
$$

This is a special case of the more general

$$
\iint_{B} f^{\prime}(z) \overline{g(z)} d x d y=\frac{i}{2} \int_{\partial B} f(z) \overline{g(z)} d \bar{z} .
$$

In formulas (5) and $\left(5^{\prime}\right)$ we assume, for simplicity, that the region $B$ has a boundary $\partial B$ that is composed of a finite number of analytic arcs, and that the functions $f(z)$ and $g(z)$ are regular in the closure of $B$.

Let the vertices of the triangle $T$ in counter-clockwise order be $z_{1}, z_{2}, z_{3}$. From (5) we obtain

$$
\begin{aligned}
\frac{2}{i} \iint_{T} f^{\prime \prime}(z) d x d y & =\int_{\partial T} f^{\prime}(z) d \bar{z} \\
& =\int_{z_{1}}^{z_{2}} f^{\prime}(z) d \bar{z}+\int_{z_{2}}^{z_{3}} f^{\prime}(z) d \bar{z}+\int_{z_{3}}^{z_{1}} f^{\prime}(z) d \bar{z}
\end{aligned}
$$

The three contour integrals are taken along the sides of the triangle $T_{1}, T_{2}, T_{3}$ respectively. Now along $T_{1}$ we have from $\left(2^{\prime}\right)$,

$$
\bar{z}=A_{1} z+B_{1}, \quad A_{1}=\frac{\bar{z}_{1}-\bar{z}_{2}}{z_{1}-z_{2}}, \quad B_{1}=\frac{z_{1} \bar{z}_{2}-z_{2} \bar{z}_{1}}{z_{1}-z_{2}} .
$$

Therefore along $T_{1}, d \bar{z}=A_{1} d z$. Hence,

$$
\left(T_{1}\right) \int_{z_{1}}^{z_{2}} f^{\prime}(z) d \bar{z}=A_{1} \int_{z_{1}}^{z_{2}} f^{\prime}(z) d z=A_{1}\left(f\left(z_{1}\right)-f\left(z_{2}\right)\right) .
$$


Similar formulas hold for $T_{2}$ and $T_{3}$ and therefore,

$$
\begin{aligned}
\frac{2}{i} \iint_{T} f^{\prime \prime}(z) d x d y & =A_{1}\left(f\left(z_{1}\right)-f\left(z_{2}\right)\right)+A_{2}\left(f\left(z_{2}\right)-f\left(z_{3}\right)\right)+A_{3}\left(f\left(z_{3}\right)-f\left(z_{1}\right)\right) \\
& =\left(A_{3}-A_{1}\right) f\left(z_{1}\right)+\left(A_{1}-A_{2}\right) f\left(z_{2}\right)+\left(A_{2}-A_{3}\right) f\left(z_{3}\right) .
\end{aligned}
$$

From (7) and (4),

$$
\begin{aligned}
A_{3}-A_{1} & =\frac{\bar{z}_{3}-\bar{z}_{1}}{z_{3}-z_{1}}-\frac{\bar{z}_{1}-\bar{z}_{2}}{z_{1}-z_{2}}=\frac{1}{\left(z_{1}-z_{2}\right)\left(z_{1}-z_{3}\right)}\left|\begin{array}{ccc}
z_{1} & \bar{z}_{1} & 1 \\
z_{2} & \bar{z}_{2} & 1 \\
z_{3} & \bar{z}_{3} & 1
\end{array}\right| \\
& =\frac{4}{i} \frac{A\left(z_{1}, z_{2}, z_{3}\right)}{\left(z_{1}-z_{2}\right)\left(z_{1}-z_{3}\right)}=\frac{4 A}{i\left(z_{1}-z_{2}\right)\left(z_{1}-z_{3}\right)} .
\end{aligned}
$$

Similar formulas hold for $A_{1}-A_{2}$ and $A_{2}-A_{3}$, and their insertion in (9) leads to (1).

3. Some Applications and Extensions. Write $f(z) \equiv\left(z-z_{1}\right)\left(z-z_{2}\right)\left(z-z_{3}\right)$. Then, $f^{\prime \prime}(z)=6 z-2 S_{1}$ where $S_{1}=z_{1}+z_{2}+z_{3}$. Hence, from (1),

$$
\frac{1}{2 A} \iint_{T}\left(6 z-2 S_{1}\right) d x d y=0 .
$$

Therefore,

$$
\iint_{T} z d x d y=\frac{S_{1}}{3} \iint_{T} d x d y=\frac{S_{1} A}{3}
$$

or

$$
\frac{1}{A} \iint_{T} z d x d y=\frac{1}{3}\left(z_{1}+z_{2}+z_{3}\right),
$$

the formula for the center of gravity of a triangle.

By taking the real or imaginary parts of (1), we can obtain the integrals of harmonic functions over triangles. For example, the product of inertia can be obtained in this way. We have

$$
\begin{aligned}
\iint_{T} x y d x d y & =\frac{1}{2} \operatorname{Im} \iint_{T} z^{2} d x d y=\frac{1}{24} \operatorname{Im} \iint_{T}\left(z^{4}\right)^{\prime \prime} d x d y \\
& =\frac{A}{12} \operatorname{Im}\left(\left|\begin{array}{lll}
1 & 1 & 1 \\
z_{1} & z_{2}{ }^{4} & z_{3} \\
z_{1}{ }^{4} & z_{2}{ }^{4} & z_{3}{ }^{4}
\end{array}\right|:\left|\begin{array}{lll}
1 & 1 & 1 \\
z_{1} & z_{2} & z_{3} \\
z_{1}{ }^{2} & z_{2}{ }^{2} & z_{3}{ }^{2}
\end{array}\right|\right) \\
& =\frac{A}{12} \operatorname{Im}\left[\left(z_{1}+z_{2}+z_{3}\right)^{2}-\left(z_{1} z_{2}+z_{1} z_{3}+z_{2} z_{3}\right)\right] .
\end{aligned}
$$

Hence, if the center of gravity of $T$ is at the origin: $z_{1}+z_{2}+z_{3}=0$, we obtain the neat formula 
(12)

$$
\text { Product of inertia of } T=\iint_{T} x y d x d y=-\frac{A}{12} \operatorname{Im}\left(z_{1} z_{2}+z_{1} z_{3}+z_{2} z_{3}\right) \text {. }
$$

Formula (1) may be extended to polygons.

Lemma. Let $z_{1}, z_{2}, \cdots, z_{n}$ designate the vertices of a polygon $P$. Then we can find constants $a_{1}, \cdots, a_{n}$ depending upon $z_{1}, \cdots, z_{n}$ but independent of $f$, such that for all $f$ regular in the closure of $P$,

$$
\iint_{P} f^{\prime \prime}(z) d x d y=\sum_{i=1}^{n} a_{i} f\left(z_{i}\right)
$$

If $r \geqq n$, and $z_{n+1}, z_{n+2}, \cdots, z_{r}$ are additional points distinct from $z_{1}, z_{2}, \cdots, z_{n}$, and if there are constants $b_{1}, \cdots, b_{r}$ which depend only upon $z_{1}, \cdots, z_{r}$ such that

$$
\iint_{P} f^{\prime \prime}(z) d x d y=\sum_{i=1}^{r} b_{i} f\left(z_{i}\right)
$$

for all $f$ regular in the closure of $P$, then

$$
b_{1}=a_{1}, \cdots, b_{n}=a_{n} \text {, and } b_{n+1}=b_{n+2}=\cdots=b_{r}=0 .
$$

Proof. The polygon $P$ may be decomposed into a finite number of triangles $T_{1}, T_{2}, \cdots, T_{s}$ such that vertices of these triangles are among the points $z_{1}, \cdots$, $z_{n}$. Write

$$
\iint_{P} f^{\prime \prime} d x d y=\sum_{i=1}^{s} \iint_{\boldsymbol{T}_{i}} f^{\prime \prime} d x d y
$$

and apply (1) to each $T_{i}$. This proves (13).

From (14) and (13) we have

$$
\sum_{i=1}^{n}\left(a_{i}-b_{i}\right) f\left(z_{i}\right)+\sum_{i=n+1}^{r} b_{i} f\left(z_{i}\right)=0
$$

so that with an obvious notation, $\sum_{i=1}^{r} c_{i} f\left(z_{i}\right)=0$ for all $f$ regular in the closure of $P$. In particular, for $f(z) \equiv z^{q}, q=0,1, \cdots, r-1$, we have $\sum_{i=1}^{r} c_{i} z_{i}^{q}=0$. Since the Vandermonde determinant $\left|z_{i}{ }^{a}\right| \neq 0$, it follows that $c_{i}=0, i=1,2, \cdots, r$ and (15) follows.

What we are asserting in (15) is, essentially, the linear independence of the functionals $L_{i}(f)=f\left(z_{i}\right), i=1,2, \cdots, r$.

We now give an explicit representation of the formula (13).

Theorem. With the notation as in the lemma,

$$
\begin{aligned}
& \iint_{\boldsymbol{P}} f^{\prime \prime}(z) d x d y \\
& \quad=-\left|\begin{array}{ccccc}
0 & f\left(z_{1}\right) & f\left(z_{2}\right) & \cdots & f\left(z_{n}\right) \\
\mu_{0} & 1 & 1 & & 1 \\
\vdots & \vdots & \vdots & & \vdots \\
\mu_{n-1} & z_{1}{ }^{n-1} & z_{n}{ }^{n-1} & \cdots & z_{n}{ }^{n-1}
\end{array}\right|:\left|\begin{array}{cccc}
1 & 1 & \cdots & 1 \\
z_{1} & z_{2} & \cdots & z_{n} \\
\vdots & \vdots & & \vdots \\
z_{1}{ }^{n-1} & z_{2}{ }^{n-1} & \cdots & z_{n}{ }^{n-1}
\end{array}\right|
\end{aligned}
$$


where

$$
\begin{gathered}
\mu_{k}=\iint_{P}\left(z^{k}\right)^{\prime \prime} d x d y=k(k-1) \iint_{P} z^{k-2} d x d y, \quad k \geqq 2, \\
\mu_{0}=\mu_{1}=0 .
\end{gathered}
$$

Proof. This comes from inserting $f \equiv 1, f \equiv z, \cdots, f=z^{n-1}$ in (13) and solving the resulting system.

A second representation is the following. For simplicity, we formulate it for a convex polygon.

TheOREM. Let $z_{1}, z_{2}, \cdots, z_{n}$ designate the vertices in counter-clockwise order of a convex polygon $P$. Write $z_{0}=z_{n}$ and $z_{n+1}=z_{1}$. Then, for all $f(z)$ regular in the closure of $P$,

$$
\iint_{P} f^{\prime \prime}(z) d x d y=2 \sum_{i=1}^{n} \frac{A\left(z_{i-1}, z_{i}, z_{i+1}\right)}{\left(z_{i}-z_{i-1}\right)\left(z_{i}-z_{i+1}\right)} f\left(z_{i}\right) .
$$

Proof. For fixed $i$, the polygon $P$ may be decomposed into the triangle $T_{i}$ whose vertices are $z_{i-1}, z_{i}$, and $z_{i+1}$ plus a polygon $P_{i}$ whose vertices are all the $z$ 's save $z_{i}$. Hence, by (13) and (1),

$$
\iint_{P} f^{\prime \prime}(z) d x d y=\frac{2 A\left(z_{i-1}, z_{i}, z_{i+1}\right)}{\left(z_{i}-z_{i-1}\right)\left(z_{i}-z_{i+1}\right)} f\left(z_{i}\right)+\sum_{j=1 ; j \neq i}^{n} d_{j} f\left(z_{j}\right) .
$$

In view of the lemma, a similar coefficient attaches to each vertex.

Corollary.

$$
\begin{aligned}
\sum_{i=1}^{n} \frac{A\left(z_{i-1}, z_{i}, z_{i+1}\right)}{\left(z_{i}-z_{i-1}\right)\left(z_{i}-z_{i+1}\right)} & =0, \\
\sum_{i=1}^{n} \frac{A\left(z_{i-1}, z_{i}, z_{i+1}\right)}{\left(z_{i}-z_{i-1}\right)\left(z_{i}-z_{i+1}\right)} z_{i} & =0, \\
\sum_{i=1}^{n} \frac{A\left(z_{i-1}, z_{i}, z_{i+1}\right)}{\left(z_{i}-z_{i-1}\right)\left(z_{i}-z_{i+1}\right)} z_{i}{ }^{2} & =\text { area of } P .
\end{aligned}
$$

Proof. Insert $f(z) \equiv 1, f(z) \equiv z, f(z) \equiv z^{2}$ in (18).

Corollary. Let the point $z^{*}$ lie inside the convex polygon $P$ whose vertices are $z_{1}, z_{2}, \cdots, z_{n}$. Then,

$$
\sum_{i=1}^{n} \frac{A\left(z_{i}, z^{*}, z_{i+1}\right)}{\left(z^{*}-z_{i}\right)\left(z^{*}-z_{i+1}\right)}=0
$$

Proof. Decompose $P$ into triangles whose vertices are $z_{i}, z^{*}, z_{i+1}$. In view of (1), $\iint_{P} f^{\prime \prime} d x d y$ can be expressed as a fixed linear combination of $f\left(z_{1}\right), \cdots, f\left(z_{n}\right)$, $f\left(z^{*}\right)$.

In this linear combination, the coefficient of $f\left(z^{*}\right)$ will be, according to (1),

$$
\sum_{i=1}^{n} \frac{2 A\left(z_{i}, z^{*}, z_{i+1}\right)}{\left(z^{*}-z_{i}\right)\left(z^{*}-z_{i+1}\right)}
$$

But by (13), $\iint_{P} f^{\prime \prime} d x d y$ can be expressed as a fixed linear combination of $f\left(z_{1}\right)$, 
$\cdots, f\left(z_{n}\right)$. Hence, by the lemma, the coefficient of $f\left(z^{*}\right)$ must vanish and this leads to (23).

It is interesting to notice, that in the case $n=3$, equation (23) is equivalent to

$$
z^{*}=z_{1} \frac{A\left(z^{*}, z_{2}, z_{3}\right)}{A\left(z_{1}, z_{2}, z_{3}\right)}+z_{2} \frac{A\left(z_{1}, z^{*}, z_{3}\right)}{A\left(z_{1}, z_{2}, z_{3}\right)}+z_{3} \frac{A\left(z_{1}, z_{2}, z^{*}\right)}{A\left(z_{1}, z_{2}, z_{3}\right)} .
$$

The coefficients of $z_{1}, z_{2}, z_{3}$ in this formulae are the barycentric coordinates of $z^{*}$ with respect to $z_{1}, z_{2}, z_{3}$. (See e.g., Deaux [4].)

4. Further Developments. In order to obtain complex formulas for integrals of the type $\iint_{T} g(x, y) d x d y$ where $g$ is an analytic function of the two real variables $x$ and $y$, we can write

$$
g(x, y)=g\left(\frac{1}{2}(z+\bar{z}), \frac{1}{2 i}(z-\bar{z})\right) .
$$

Expansions of $g$ in power series now suggest that one should study integrals of the form $\iint_{T} \bar{z}^{n} f(z) d x d y$. We begin with $n=1$.

Theorem. Set $z_{0}=z_{3}$ and $z_{4}=z_{1}$. Then if $f(z)$ is regular in the closure of $T$,

$$
\begin{aligned}
\frac{1}{2 A} \iint_{T} \bar{z} f^{\prime \prime \prime}(z) d x d y= & 2 \sum_{i=1}^{3} \frac{\operatorname{Re}\left[\left(z_{i}-z_{i-1}\right)\left(\bar{z}_{i}-\bar{z}_{i-1}\right)\right]}{\left(z_{i}-z_{i-1}\right)^{2}\left(z_{i}-z_{i+1}\right)^{2}} f\left(z_{i}\right) \\
& +\sum_{i=1}^{3} \frac{\bar{z}_{i}}{\left(z_{i}-z_{i-1}\right)\left(z_{i}-z_{i+1}\right)} f^{\prime}\left(z_{i}\right) .
\end{aligned}
$$

Proof. Designate the sides of $T$, properly oriented, by $T_{1}, T_{2}, T_{3}$. By $\left(5^{\prime}\right)$ we have

$$
\iint_{T} \bar{z} f^{\prime \prime \prime}(z) d x d y=\frac{i}{2} \int_{T_{1}+T_{2}+T_{3}} \bar{z} f^{\prime \prime}(z) d \bar{z} .
$$

Work on $T_{1}$ as a prototype:

$$
\begin{aligned}
& \frac{i}{2} \int_{T_{1}} \bar{z} f^{\prime \prime}(z) d \bar{z}=\frac{i}{2} \int_{z_{1}}^{z_{2}} f^{\prime \prime}(z)\left(A_{1} z+B_{1}\right) A_{1} d z \\
& =\frac{i}{2}\left[\left[f^{\prime}(z) A_{1}\left(A_{1} z+B_{1}\right)\right]_{z_{1}}^{z_{2}}-A_{1}^{2} \int_{z_{1}}^{z_{2}} f^{\prime}(z) d z\right] \\
& =\frac{i}{2}\left[A_{1} \bar{z}_{2} f^{\prime}\left(z_{2}\right)-A_{1} \bar{z}_{1} f^{\prime}\left(z_{1}\right)\right]+\frac{i}{2} A_{1}{ }^{2}\left[f\left(z_{1}\right)-f\left(z_{2}\right)\right] \text {. }
\end{aligned}
$$

The first bracket holds since on $T_{1}, \bar{z}=A_{1} z+B$. Hence, $\bar{z}_{1}=A_{1} z_{1}+B_{1}$, $\bar{z}_{2}=A_{1} z_{2}+B_{2}$.

Similarly,

$$
\frac{i}{2} \int_{T_{1}} \bar{z} f^{\prime \prime}(z) d \bar{z}=\frac{i}{2}\left[A_{2} \bar{z}_{3} f^{\prime}\left(z_{3}\right)-A_{2} \bar{z}_{2} f^{\prime}\left(z_{2}\right)\right]+\frac{i}{2} A_{2}^{2}\left[f\left(z_{2}\right)-f\left(z_{3}\right)\right]
$$

and a corresponding expression holds for the integral along $T_{3}$. Forming the sum along the three sides, and picking out, for example, the coefficients of $f\left(z_{2}\right)$ and $f^{\prime}\left(z_{2}\right)$, we have as the contribution from the point $z_{2}$ : 


$$
\frac{i}{2}\left(A_{1}-A_{2}\right)\left[\bar{z}_{2} f^{\prime}\left(z_{2}\right)+\left(A_{2}+A_{1}\right) f\left(z_{2}\right)\right]
$$

From (10),

$$
A_{1}-A_{2}=\frac{4 A}{i\left(z_{2}-z_{1}\right)\left(z_{2}-z_{3}\right)}
$$

Also,

$$
A_{2}+A_{1}=\frac{\bar{z}_{2}-\bar{z}_{3}}{z_{2}-z_{3}}+\frac{\bar{z}_{1}-\bar{z}_{2}}{z_{1}-z_{2}}=\frac{2 \operatorname{Re}\left[\left(z_{2}-z_{1}\right)\left(\bar{z}_{2}-\bar{z}_{3}\right)\right]}{\left(z_{2}-z_{3}\right)\left(z_{2}-z_{1}\right)}
$$

Formula (25) now follows.

As an application of this formula, select $f(z) \equiv z\left(z-z_{1}\right)\left(z-z_{2}\right)\left(z-z_{3}\right)$. Then $f^{\prime \prime \prime}(z)=24 z-6 S_{1}, S_{1}=z_{1}+z_{2}+z_{3}$. Now, $f^{\prime}\left(z_{1}\right)=z_{1}\left(z_{1}-z_{2}\right)\left(z_{1}-z_{3}\right)$, etc., hence from (25),

$$
\frac{1}{2 A} \iint_{T} \bar{z}\left(24 z-6 S_{1}\right) d x d y=\bar{z}_{1} z_{1}+\bar{z}_{2} z_{2}+\bar{z}_{3} z_{3} .
$$

Inserting (11) in (27), we obtain a complex formula for the moment of inertia of a triangle about an axis perpendicular to the triangle and passing through the origin:

$$
\iint_{T}|z|^{2} d x d y=\frac{A}{12}\left[\left|z_{1}\right|^{2}+\left|z_{2}\right|^{2}+\left|z_{3}\right|^{2}+\left|z_{1}+z_{2}+z_{3}\right|^{2}\right] .
$$

If the center of gravity of the triangle is at the origin, we obtain the neat formula

$$
\iint_{T}|z|^{2} d x d y=\frac{A}{12}\left[\left|z_{1}\right|^{2}+\left|z_{2}\right|^{2}+\left|z_{3}\right|^{2}\right] .
$$

The generalization of this theorem is as follows:

Theorem. Given a triangle $T$ we can find constants $a_{i j}$ depending upon $z_{1}, z_{2}, z_{3}$ such that

$$
\iint_{T} \bar{z}^{m} f^{(m+2)}(z) d x d y=\sum_{i=0}^{m} \sum_{j=1}^{3} a_{i j} f^{(i)}\left(z_{j}\right)
$$

for all $f$ regular in the closure of $T$.

Proof. Use (5) and proceed as before.

Formula (29) may be regarded as a kind of complex three point Taylor expression with exact remainder.

5. Complex Integration over Lunes. Designate the common part of the two circles

$$
\left.\begin{array}{l}
C_{1}:|z-\sigma i|^{2}=1+\sigma^{2} \\
C_{2}:|z+\sigma i|^{2}=1+\sigma^{2}
\end{array}\right\} \quad \sigma>0, \quad i=\sqrt{-1}
$$

by $L$. 
These circles both pass through $z= \pm 1$ and their centers are at $z=\sigma i$ and $z=-\sigma i$ respectively. On $L_{1}$, the lower boundary of $L$, we have

$$
\bar{z}=S_{1}(z)=\frac{1+\sigma^{2}}{z-\sigma i}-\sigma i
$$

while on $L_{2}$, the upper boundary, we have

$$
\bar{z}=S_{2}(z)=\frac{1+\sigma^{2}}{z+\sigma i}+\sigma i
$$

If $f(z)$ is regular in the closure of $L$, then by $\left(5^{\prime}\right)$ we have

$$
\frac{2}{i} \iint_{L} f(z) \bar{z}^{n} d x d y=\iint_{L_{1}+L_{2}} F(z) \bar{z}^{n} d \bar{z}
$$

where $F(z)$ is an indefinite integral of $f(z)$. Now,

$$
\begin{aligned}
\int_{L_{1}} F(z) \bar{z}^{n} d \bar{z} & =\int_{L_{1}} F(z) S_{1}^{n}(z) S_{1}{ }^{\prime}(z) d z \\
& =\left.F(z) \frac{S_{1}^{n+1}(z)}{n+1}\right|_{-1} ^{+1}-\frac{1}{n+1} \int_{L_{1}} f(z) S_{1}^{n+1}(z) d z
\end{aligned}
$$

In view of the analyticity of $f$ and $S_{1}$, we may take this last integral along the $x$ axis, so that

$$
\begin{aligned}
(n+1) \int_{L_{1}} F(z) \bar{z}^{n} d \bar{z}= & F(1){S_{1}}^{n+1}(1)-F(-1){S_{1}}^{n+1}(-1) \\
& -\int_{-1}^{+1} f(x){S_{1}}^{n+1}(x) d x
\end{aligned}
$$

Similarly,

$$
\begin{aligned}
(n+1) \int_{L_{2}} F(z) \bar{z}^{n} d \bar{z}= & F(-1){S_{2}}^{n+1}(-1)-F(1){S_{2}}^{n+1}(1) \\
& -\int_{+1}^{-1} f(x){S_{2}}^{n+1}(x) d x
\end{aligned}
$$

Since $S_{1}(1)=S_{2}(1)=1, S_{1}(-1)=S_{2}(-1)=-1$,

$$
\frac{2}{i} \iint_{L} f(z) \bar{z}^{n} d x d y=\frac{1}{n+1} \int_{-1}^{+1} f(x)\left({S_{2}}^{n+1}(x)-{S_{1}}^{n+1}(x)\right) d x .
$$

This reduces the complex double integral to a single real integral of the usual sort. The particular values $n=0$ and $n=1$ lead to the formulas

$$
\begin{aligned}
\iint_{L} f(z) d x d y & =\sigma \int_{-1}^{+1} f(x) \frac{1-x^{2}}{\sigma^{2}+x^{2}} d x \\
\iint_{L} \bar{z} f(z) d x d y & =\sigma\left(1+\sigma^{2}\right) \int_{-1}^{+1} f(x) \frac{x\left(1-x^{2}\right)}{\left(x^{2}+\sigma^{2}\right)^{2}} d x .
\end{aligned}
$$


In a paper that follows, application will be made of equations (1) and (33) to "simple" quadratures.

The author wishes to thank Richard DeMar for a number of useful discussions.

Added in proof. The author wishes to thank Dr. Matthew P. Gaffney for calling my attention to Helmut Grunsky, "Eine Funktionentheoretische Integralformel," Math. Z. v. 63 , 1955, p. 320-323, where formula (1) will also be found.

Division of Applied Mathematics

Brown University

Providence, Rhode Island

1. P. Davis, "On simple quadratures," Proc. Amer. Math. Soc., v. 4, 1953, p. 127-136.

2. I. J. Schom ne RG, Approximations: Theory and Practice, Notes by L. H. Lange on a series of lectures at Stanford University, Stanford, Calif., 1955 .

3. P. DAvis \& H. Pollak, "On the analytic continuation of mapping functions," Trans. Amer. Math. Soc., v. 87, 1958, p. 198-225. 1956.

4. Roland DeAux, Introduction to the Geometry of Complex Numbers, Ungar, New York, 